\title{
Longevity in mammals: lost genes as a determinant
}

\author{
A.V. Seliverstov ${ }^{1 *}$, G.A. Shilovsky ${ }^{2}$, L.I. Rubanov ${ }^{1}$, O.A. Zverkov ${ }^{1}$, V.A. Lyubetsky ${ }^{1,2}$ \\ ${ }^{1}$ Institute for Information Transmission Problems of the Russian Academy of Sciences \\ (Kharkevich Institute), Moscow, Russia \\ ${ }^{2}$ Lomonosov Moscow State University, Moscow, Russia \\ *e-mail:slvstv@iitp.ru
}

Key words: lifespan, longevity, neoteny, naked mole-rat, synteny, orthology, gene loss

Motivation and Aim: The genetic propensity of certain species for longevity and antiageing is a challenging problem of vertebrate biology etc. Following our general hypothesis on the evolutionary significance of gene loss, we tried to identify lost genes in mammals with a greater lifespan than could be expected from their body size: mole rats, and primates (including the naked mole rat, little brown bat, capuchin monkeys, gibbons, western gorilla, bonobo, chimpanzee, and human). Species living under different conditions were analyzed to reduce the habitat impact on the phenomenon studied.

Methods and Algorithms: This study relies on our previous bioinformatics method experimentally approved while testing the hypothesis for regeneration potential and endbrain development. The method takes into account the similarity and local synteny of genes and generates amazingly short lists of lost genes.

Results: The list of lost mouse genes includes Abca14, Ace3, Csn 1s2a, Gm17416, Hist1h2af, Prss 43, Slc6a21, Smpd5, Spint5 (a Kunitz type 5 serine protease inhibitor), Ttc41, Wap, Wfdc16 (a peptidase inhibitor affecting cell proliferation), 2310003L06Rik expressed in the tongue, and certain vomeronasal and olfactory receptor genes.

Discussion: The mouse Smpd5 gene encodes the neutral sphingomyelinase responsible for breaking down sphingomyelin, a critical factor of the integrated mitochondrial reticulum formation in the skeletal muscles; and its development is notably decelerated in the naked mole rat relative to mouse. A homologous sphingomyelinase is involved in apoptosis initiation through the formation of pores in the mitochondrial membrane. The mouse $\operatorname{Tm} \operatorname{bim} 7$ encodes a transmembrane protein, a putative apoptosis inhibitor; it is actively expressed in the mouse testis and corresponds to the human TMBIM7P pseudogene. The gene corresponds to the ENSGL00000028944 pseudogene in the female naked mole rat; without regard to synteny, the gene aligns with the TMBIM6 gene, which is overexpressed in certain cancer types and suppresses Bax-induced apoptosis.

Conclusion: The revealed mouse genes demonstrate specific expression in reproductionassociated tissues, which agrees with the Williams' hypothesis concerning the reallocation of physiological recourses of an organism between the self-maintenance and reproduction. The loss of some revealed vomeronasal and olfactory receptor genes in human and naked mole rat conforms to special anatomical features. We suggest that the loss of certain genes in evolution is a determinant of lifespan elongation and ageing deceleration including neoteny. Availability: http:/lab6.iitp.ru/en/lossgainrsl/ Acknowledgements: The research was supported by the Russian Science Foundation, project No. 14-50-00150. The research was carried out using supercomputers at Joint Supercomputer Center of the Russian Academy of Sciences (JSCC RAS). 